\title{
Ultrafast excited-state charge-transfer dynamics in laccase type I copper site
}

\author{
Ines Delfino ${ }^{\mathrm{a}, *}$, Daniele Viola $^{\mathrm{b}}$, Giulio Cerullo $^{\mathrm{b}}$, Maria Lepore $^{\mathrm{c}}$ \\ a Dipartimento di Scienze Ecologiche e Biologiche, Università della Tuscia, Largo dell'Università snc, I-01100 Viterbo, Italy \\ b IFN-CNR, Dipartimento di Fisica, Politecnico di Milano, Piazza Leonardo da Vinci 32, I-20133 Milano, Italy \\ c Dipartimento di Medicina Sperimentale, Seconda Università di Napoli, Via Costantinopoli 16, I-80100 Napoli, Italy
}

\section{H I G H L I G H T S}

- T1Cu MLCT in POXC is described by ultrafast pump-probe spectroscopy

- The first step of the charge-transfer in POXC occurs within 375 fs

- Ground-state coherent oscillations are compared to Resonance Raman features

- The trigonal T1 Cu site geometry hypothesized for POXC is confirmed by the results

\section{G R A P H I C A L A B S T R A C T}
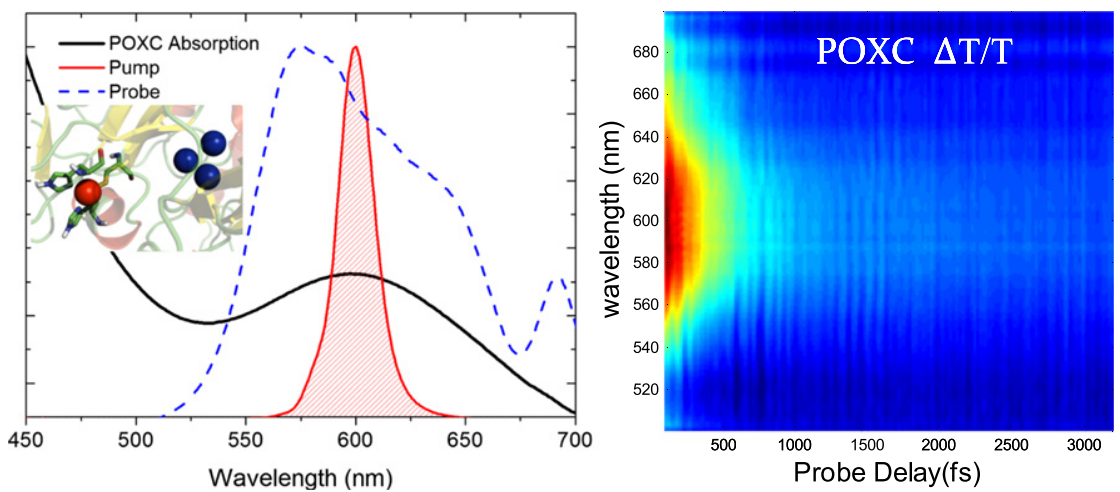

\section{A B S T R A C T}

Femtosecond pump-probe spectroscopy was used to investigate the excited state dynamics of the 11 copper site of laccase from Pleurotus ostreatus, by exciting its $600 \mathrm{~nm}$ charge transfer band with a 15 -fs pulse and probing over a broad range in the visible region. The decay of the pump-induced ground-state bleaching occurs in a single step and is modulated by clearly visible oscillations. Global analysis of the two-dimensional differential transmission map shows that the excited state exponentially decays with a time constant of $375 \mathrm{fs}$, thus featuring a decay rate slower than those occurring in quite all the investigated T1 copper site proteins. The ultrashort pump pulse induces a vibrational coherence in the protein, which is mainly assigned to ground state activity, as expected in a system with fast excited state decay. Vibrational features are discussed also in comparison with the traditional resonance Raman spectrum of the enzyme. The results indicate that both excited state dynamics and vibrational modes associated with the $\mathrm{T} 1 \mathrm{Cu}$ laccase charge transfer have main characteristics similar to those of all the T1 copper site-containing proteins. On the other hand, the differences observed for laccase from $P$. ostreatus further confirm the peculiar hypothesized trigonal T1 Cu site geometry.

(c) 2015 Elsevier B.V. All rights reserved.

\footnotetext{
* Corresponding author.

E-mail address: delfino@unitus.it (I. Delfino).
}

\section{Introduction}

Laccases are the largest group of blue multicopper oxidases (MCOs) and represent one of the six classes of enzymes that couple the fourelectron reduction of $\mathrm{O}_{2}$ to $\mathrm{H}_{2} \mathrm{O}$ with four one-electron oxidations of 
substrates [1,2]. These oxidation and reduction processes are obtained using four $\mathrm{Cu}$ atoms distributed against three sites. $\mathrm{Cu}$ sites are classified into three types according to their spectroscopic properties: type 1 (T1) or blue $\mathrm{Cu}$, type 2 (T2) or normal $\mathrm{Cu}$, and type 3 (T3) or coupled binuclear $\mathrm{Cu}$ sites [1-4]. A plethora of comparative studies have established that the copper coordination of the T2 and T3 centers is very similar among the MCOs while there are differences in the T1 Cu ligation. In fungal laccases $\mathrm{T} 1 \mathrm{Cu}$ has a planar triangular coordination with the sulfur atom of a cysteine and with the $\mathrm{N}_{1}$ nitrogen of two histidines, giving rise to a very peculiar trigonal geometry which differs from the tetrahedrically coordinated T1 copper of the other MCOs in which the copper atom is ligated also to a fourth axial ligand (usually a methionine). In fact laccases isolated from fungi possess a leucine or phenylalanine rather than a methionine at the axial position [3,5-8], but these amino acids do not contain functional groups that can ligate to the copper atom and their influence on the T1 Cu site is still under debate [9]. The lack of the fourth axial ligand in laccases is considered as an important factor determining the higher values of redox potential displayed by laccases in comparison with the other MCOs [1]. The T1 $\mathrm{Cu}$ site geometry is responsible for the absorption of the enzyme in the red due to an intense Cys-S to $\mathrm{Cu}$ (II) charge-transfer (CT) band, called ligand-to-metal charge transfer (LMCT), with a maximum at about $600 \mathrm{~nm}[8,11]$. In fungal laccases the copper atoms of T2 and T3 sites are arranged in a triangular fashion (forming a trinuclear cluster, TNC), as consistently observed in MCOs, and coordinated to a strongly conserved pattern of four His-X-His motifs. This bridge provides an efficient super exchange pathway for rapid intramolecular electron transfer (ET) from the T1 Cu to the trinuclear cluster. In fact, the $\mathrm{T} 1 \mathrm{Cu}$ is the primary electron acceptor site in a laccase catalyzed reaction, where the four single-electron oxidations of a reducing substrate occur. The electrons are then shuttled through the highly conserved His-Cys-His tripeptide to the TNC, where $\mathrm{O}_{2}$ is reduced to water $[2,3]$.

Ultrafast resonant pump-probe spectroscopy is a powerful tool to investigate the charge transfer processes in copper proteins and the relationship between enzyme structure and functionalities [11-19]. In these experiments, the protein is excited in resonance with the absorption band by an ultrashort pump pulse that creates a population in the excited electronic state and vibrational coherences in both the ground and excited states. A delayed probe pulse then interrogates the sample, and its pump-induced transmission change (differential transmission, $\Delta \mathrm{T} / \mathrm{T}$ ) is detected. In this way excited-state population dynamics and real-time analysis of vibrational motions coupled to electronic transitions are investigated. By resonant pump-probe spectroscopy the CT dynamics occurring in T1 Cu site of various cupredoxins has been investigated [11,14-19]. The excited state dynamics of some plastocyanins has been described as a multi-step relaxation process (two-step process in spinach plastocyanin, with characteristic times of 125 and 285 fs for the first and second steps, respectively [11]; three-step deactivation for Silene pratensis plastocyanin, with time constants of 40, 90 and 250 fs [19]). At variance, a single step deactivation process (with a time constant of $270 \mathrm{fs}$ ) has been observed for plastocyanin from Synechococcus PCC7942 [18], azurin [14], and poplar plastocyanin [15], while a slightly faster single step process has been detected for the rusticyanin CT transition (time constant of $230 \mathrm{fs}$ ) [17]. The dynamics of the CT excited state in umecyanin from Horseradish root has also been described as a single step ground state population recovery process, with probe wavelength-dependent time constants (from 470 to $700 \mathrm{fs}$ ) [16]. In all the cases, the knowledge of the excited state dynamics has helped in better describing the overall CT process and the copper site geometry. Despite the successful use of resonant pump-probe spectroscopy in studying cupredoxins, no similar ultrafast study about MCOs is available until now. In fact, the only pump-probe study on a MCO is by Book et al. [20] who reported pump-probe measurements on human ceruloplasmin, following an excitation pulse centered at $770 \mathrm{~nm}$, not in resonance with protein T1 Cu LMCT band.
In this work we use ultrafast resonant pump-probe spectroscopy to study the dynamics of the first step of the charge transfer process occurring in laccases, i.e., the charge transfer taking place at the $\mathrm{T} 1 \mathrm{Cu}$ site. In our experiments, Pleurotus ostreatus laccase (POXC) [21] in the solution has been excited by a 15 fs pulse resonant with the T1 Cu LMCT transition and interrogated by a broadband visible probe pulse covering the whole ground state absorption band of POXC. We have observed that the charge-transfer excited state decays to the ground state via a single-step relaxation with a time constant of 375 fs. Fourier transform (FT) analysis of the oscillatory pattern superimposed on the signal has revealed well-resolved vibrational frequencies in the $350-500 \mathrm{~cm}^{-1}$ spectral range. Almost all the observed modes have corresponding ones in the conventional resonance Raman (RR) spectrum reported here for the first time.

\section{Materials and methods}

\subsection{Sample preparation and characterization}

POXC [21] was obtained by BioPox (Napoli, Italy) in a $14 \mathrm{mg} / \mathrm{ml} \mathrm{so-}$ lution. It is a $67-\mathrm{kDa}$ fungal laccase with 4 copper atoms distributed against three sites, T1, T2 and T3 as said above. POXC, as all fungal laccases, exhibits a molecular architecture organized in three sequentially arranged cupredoxin-like domains. Each of them has a Greek key $\beta$-barrel topology, strictly related to that of small copper proteins such as azurin and plastocyanin and common to all the members of the MCO family. The T1 Cu is located in domain 3, while the TNC cluster is embedded between domains 1 and 3 with both domains providing residues for copper coordination. The 3D structure of POXC as obtained by using Pymol is shown in Fig. 1a [22].

For all measurements POXC was exchanged into $50 \mathrm{mM} \mathrm{NaSO}_{4}$ pH 6.4 buffer. Absorption measurements were performed at room temperature using a two-beam UV-VIS spectrophotometer (Jasco V-500) in the spectral region $200-800 \mathrm{~nm}$ with a $2.0 \mathrm{~nm}$ band pass using a protein concentration of $200 \mu \mathrm{M}$. A representative absorption spectrum of POXC in the visible region is shown in Fig. 1b (black line). The main band peaks at about $600 \mathrm{~nm}$ and corresponds to the T1 copper site LMCT transition between $\mathrm{S}(\mathrm{Cys}-\pi)$ and $\mathrm{Cud}_{\mathrm{x}^{2}-\mathrm{y}^{2}}$, which results from the superposition of six bands (see Fig. 1c for a representation of the corresponding energy level scheme) $[7,10]$.

The high absorbance for wavelengths shorter than $500 \mathrm{~nm}$ is due to the tail of the complex absorption spectrum of the protein, deriving from the overlap of various contributions, including the typical band due to the $\mathrm{T} 3 \mathrm{Cu}$ site peaking at about $330 \mathrm{~nm}$.

\subsection{Femtosecond pump-probe experiments}

The experimental setup used for femtosecond pump-probe spectroscopy has been described in detail elsewhere [23]. Briefly, a modelocked Ti:sapphire system with chirped pulse amplification produces $500 \mu, 150 \mathrm{fs}$ pulses at a wavelength of $790 \mathrm{~nm}$ with a $1 \mathrm{kHz}$ repetition rate. These pulses are used to pump two non-collinear optical parametric amplifiers (NOPAs), which have the capability of generating broadband visible pulses. The first NOPA produces 15 fs transform-limited pulses with a tunable center frequency; the second NOPA generates ultra-broadband visible pulses spanning the 500-700 nm wavelength range and with sub-10-fs duration. For both NOPAs the pulses are compressed by multiple reflections onto chirped dielectric mirrors, which are particularly insensitive to alignment and allow consistently short pulses to be obtained [24]. The pulses derived from the NOPAs are synchronized by a delay line and focused on the sample using reflective optics, in a standard non-collinear pump-probe configuration; an intense pulse from the first NOPA serves as the pump, while an attenuated, delayed pulse from the second NOPA probes the $\Delta \mathrm{T} / \mathrm{T}$ changes. The pump and probe pulses are superimposed on the sample in a slightly non- 

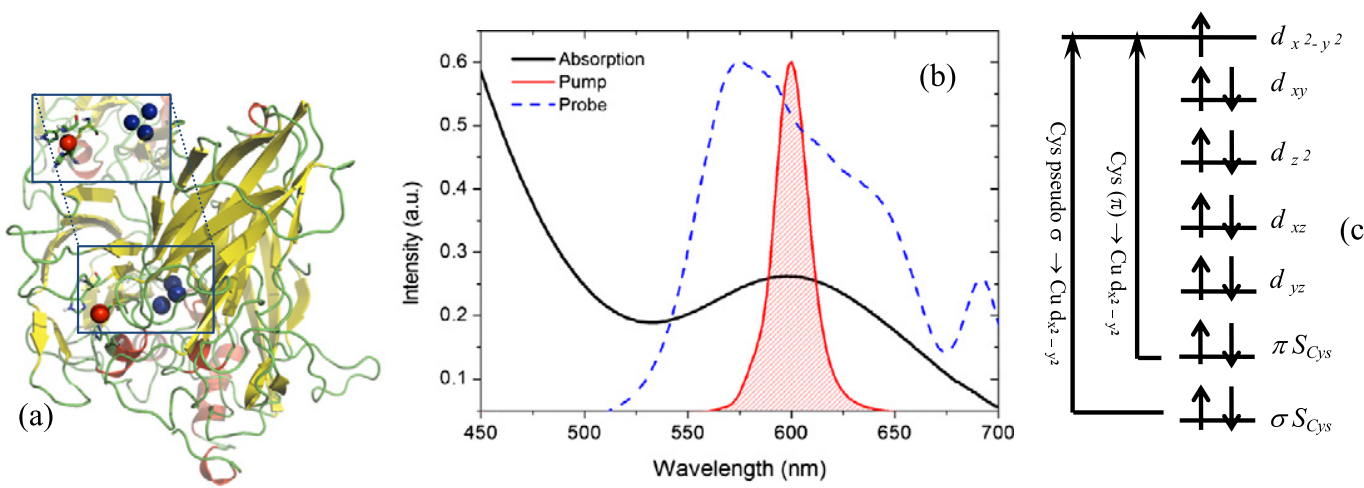

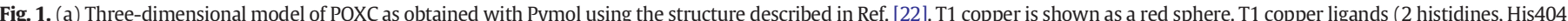

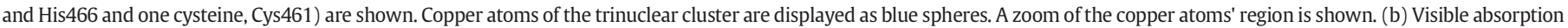

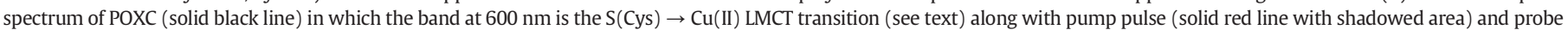

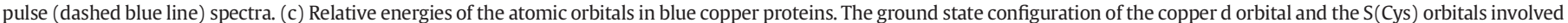
in the visible transitions are shown. The energy level gaps are not drawn to scale.

collinear geometry, and their delay is controlled by a motorized translation stage. The pump pulse is modulated at $500 \mathrm{~Hz}$ by a chopper wheel. After the sample, the probe beam is focused onto the entrance slit of a spectrometer equipped with a linear photodiode array with a fast electronics allowing a full $1 \mathrm{kHz}$ read-out rate. By recording pump-on and pump-off probe spectra, we calculate the differential transmission, $\Delta \mathrm{T} / \mathrm{T}$, signal as a function of probe wavelength $\lambda$ and pump-probe delay $\tau$ as $\Delta \mathrm{T} / \mathrm{T}(\lambda, \tau)=\left(\mathrm{T}_{\text {on }}(\lambda, \tau)-\mathrm{T}_{\text {off }}(\lambda)\right) / \mathrm{T}_{\text {off }}(\lambda)$.

For our experiments the pump pulse was tuned to $600 \mathrm{~nm}$ (Fig. 1b, red line), to achieve optimum overlap with the POXC T1 Cu LMCT absorption band, and the probe pulse was tuned in the visible range (Fig. 1b, blue dashed line). Pump and probe beams were set to parallel polarization.

POXC solutions were kept at room temperature in a home-made cuvette, employing $200 \mu \mathrm{m}$ thick fused silica windows and with an optical path of $\approx 600 \mu \mathrm{m}$. Experiments typically required $10 \mu \mathrm{l}$ of sample at a concentration of $0.64 \mathrm{mM}$. Since the average power is quite low, in the $\mu \mathrm{W}$ range, no sample degradation is expected to occur; nevertheless, the solution was replaced at the beginning of each experimental run. Excitation intensity was maintained in the linear regime, with $\Delta \mathrm{T} / \mathrm{T}$ below 5-6\%. POXC absorption spectra were taken immediately before and immediately after the pump-probe measurements to check that no photodegradation has occurred.

\subsection{Data analysis}

The two-dimensional (2D) $\Delta \mathrm{T} / \mathrm{T}$ maps were analyzed by global analysis, using an ad hoc written software based on the approach described in Ref. [25], running under Matlab (Matlab 8.0, MathWorks, Natick, Massachusetts, USA). Briefly, $\Delta \mathrm{T} / \mathrm{T}$ maps were analyzed using a parallel kinetic model ( $1|2| 3 \mid \ldots n)$, in which $n$ mono-exponential decaying processes beginning at the same time are considered. Accordingly, the wavelength-time $2 \mathrm{D} \Delta \mathrm{T}$ / T maps were fitted using a sum of $\mathrm{n}$ terms, each being the product of a 1D exponential decay function in time (time constant $\tau_{\mathrm{i}}, \mathrm{i}=1, \ldots, \mathrm{n}$ ) with a 1D spectrum (called "decayassociated differential spectrum," DADS). In this manner, the exponential decay constants were assumed to be independent of wavelength. Each exponential decay was convolved with the impulse response function (IRF), assumed as a Gaussian function of $\approx 60 \mathrm{fs}$ FWHM, to take into account the nonlinear solvent response. This procedure allowed the measured $\Delta \mathrm{T} / \mathrm{T}$ maps to be decomposed into various contributions, each describing the $\Delta \mathrm{T} / \mathrm{T}$ spectrum associated with a specific decay time. The number of terms ( $\mathrm{n}$ ) to be considered was defined by optimizing the fitting results and by a singular value decomposition approach, as suggested in Ref. [25]. Given the IRF and the signal-to-noise ratio of our data, we can estimate a time resolution of at least $30 \mathrm{fs}$.

\section{Results and discussion}

Fig. 2a shows a representative 2D $\Delta T / T(\lambda, \tau)$ map for POXC following excitation with a $15 \mathrm{fs}$ pulse tuned at $600 \mathrm{~nm}$ for the time delays $>60 \mathrm{fs}$. The signal around zero time delay is not shown since it is dominated by the non-resonant response of the buffer solution, which vanishes when pump and probe are well separated temporally [15,26]. For time delays longer than 60 fs the solvent response vanishes and we therefore detect the pure enzyme signal. It shows an increased transmission $(\Delta \mathrm{T} / \mathrm{T}>0)$ in the $540-660 \mathrm{~nm}$ probe wavelength region, which can be assigned to the superposition of photobleaching (PB) of the ground state of the T1 site LMCT transition and stimulated emission (SE) from the excited state. The $\Delta \mathrm{T} / \mathrm{T}$ signal decays on the picosecond timescale, indicating fast recovery of the ground-state population. An oscillatory pattern superimposed on this decay is observed throughout the analyzed time delay and probe wavelength range. This is assigned to vibrational coherence, created by the very short pump pulses in the excited and ground state multi-dimensional potential energy surfaces (PES) [27]. $\Delta \mathrm{T} / \mathrm{T}$ time traces extracted at different probe wavelengths, starting from $60 \mathrm{fs}$, and $\Delta \mathrm{T} / \mathrm{T}$ spectra at different time delays, shown in Fig. 2b and c, respectively, confirm the above discussed qualitative behavior.

To obtain a quantitative description of the processes giving rise to the signal, the $\Delta \mathrm{T} / \mathrm{T}$ map was analyzed by global analysis as described in the Materials and methods section, in the 0-3200 fs time range and for the 500-700 spectral range. Three parallel components were required to describe the $2 \mathrm{D} \Delta \mathrm{T} / \mathrm{T}$ map, whose characteristics are shown in Fig. 3, where the DADS (Fig. 3a) and the corresponding dynamics (Fig. 3b) are shown. The first component has a lifetime of $22 \pm 4$ fs and a DADS showing the derivative-like features expected for the cross-phase modulation coherent artifact, occurring during pumpprobe temporal superposition. This contribution can be neglected in discussing enzyme excited state dynamics. The second component has a time constant of $375 \pm 25$ fs and a DADS peaking at around $600 \mathrm{~nm}$, close to the peak of the ground state absorption, which has a maximum at around $605 \mathrm{~nm}$ (see Fig. 1b). The third component has a spectral shape similar to the first one (peaking at $600 \mathrm{~nm}$ ) with a smaller amplitude and a significant longer lifetime which results to be in the order of $9 \mathrm{ps}$, greatly exceeding the upper limit of the investigated temporal window, fixed at 3 ps. The observed almost infinite lifetime - in the investigated window - of this component makes it hardly distinguishable from a constant component. 

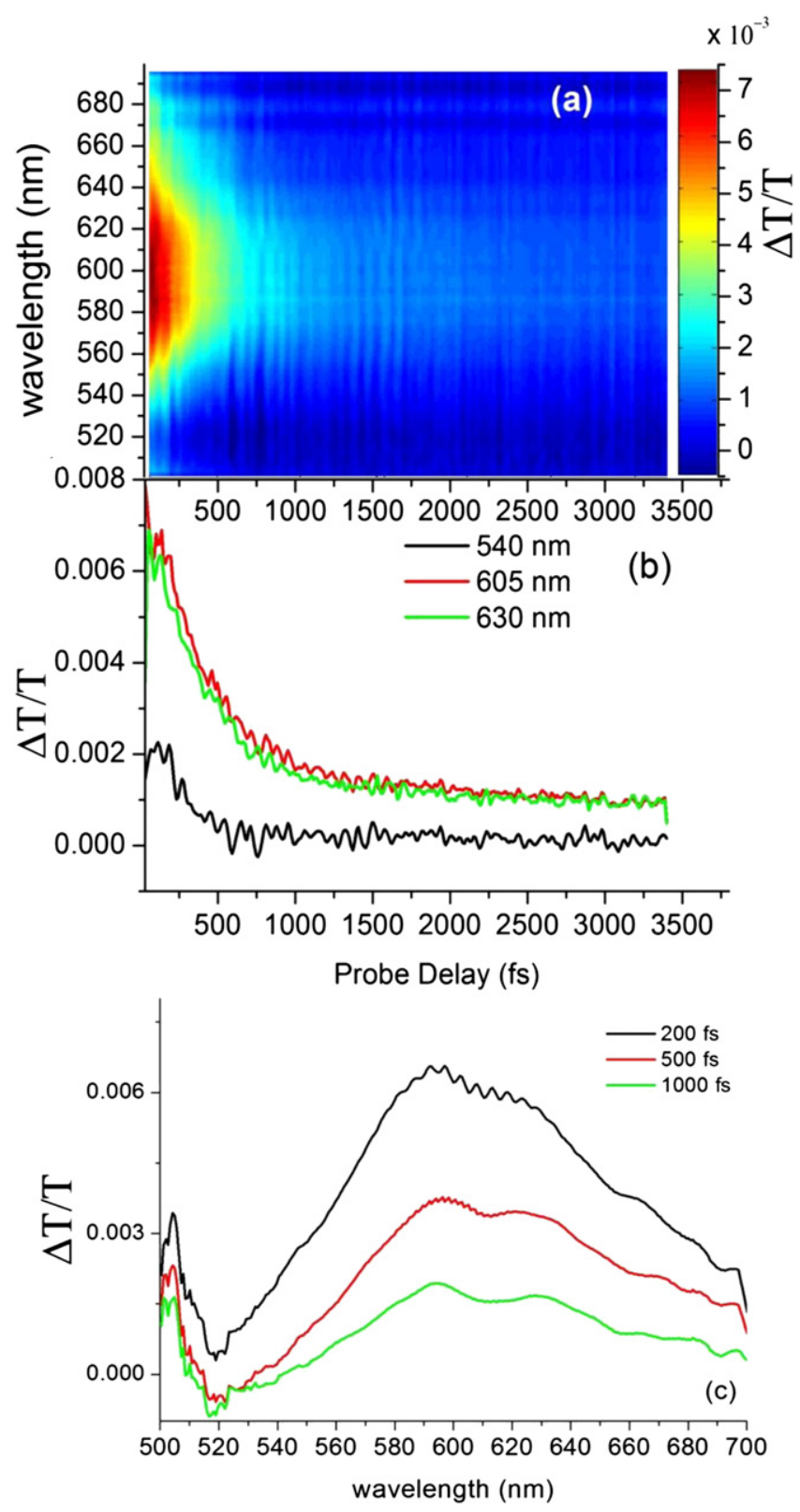

Fig. 2. (a) Two-dimensional $\Delta \mathrm{T} / \mathrm{T}$ map of POXC in the visible region following a $600-\mathrm{nm}$ pump excitation. (b) $\Delta \mathrm{T} / \mathrm{T}$ time traces extracted at specific probe wavelengths. (c) $\Delta \mathrm{T} / \mathrm{T}$ spectra extracted at specific probe delays. The oscillatory features observed in the spectra in the 580-620 nm wavelength range are assigned to spectral interference between the scattered pump beam and the probe.

Interestingly, the ratio between the amplitude of the 9-ps DADS and that of the 375-fs DADS is quite flat over the investigated spectral range and equals 0.2 (i.e., $20 \%$ ).

DADS characteristics and associated dynamics suggest that in the investigated temporal window the enzyme signal can be well described with a predominant single exponential decay (with $\tau=375 \pm 25 \mathrm{fs}$ ) plus a constant offset. This well correlates with previous experiments on $\mathrm{T} 1 \mathrm{Cu}$ site-containing proteins in which the $\Delta \mathrm{T} / \mathrm{T}$ time traces extracted from a 2D map were usually analyzed in terms of a sum of decaying exponentials plus a constant offset $[16,20]$. We can therefore conclude that, after the light-induced excitation of the LMCT T1 Cu site transition, a single deactivation process with a lifetime of $375 \pm 25$ fs occurs. The rapidly decaying exponential indicates that most of the excited state population created by the pump pulse quickly returns to the ground state (or states) in a non-radiative fashion by an ultrafast internal conversion process. In this case the positive $\Delta \mathrm{T} / \mathrm{T}$ signal is assigned to a PB process, also considering the spectral characteristics of the corresponding DADS, recalling those of the ground state absorption spectrum of the protein. Since these electronic transitions involve the movement of charge from ligand atoms to the copper center, the decay of excited state population can be likely assigned to a return charge transfer process which is called metal-to-ligand chargetransfer (MLCT). The constant offset (corresponding to the almost infinite lifetime exponential decay) could be the evidence of an amount of population (around 20\%) becoming trapped in a long-living state or on the excited-state surface, preventing the reestablishment of equilibrium on the time-scale of the pump-probe experiment, as suggested in similar studies in which a constant offset was required to describe the single wavelength time traces $[11,14-18]$. These results allow us to say that after light induced LMCT excitation in POXC a single-step deactivation process with a time constant of 375 fs (in terms of a hole transfer representation [11]: S(Cys- $\pi) \rightarrow^{375}$ fs $\mathrm{Cu} \mathrm{d}_{\mathrm{x}^{2}-\mathrm{y}^{2}}$ ) occurs; thus POXC features a deactivation process similar to that of azurin [14], some plastocyanins [15,18], umecyanin [16] and rustycianin [17]. In fact, by using the analysis of single time traces detected at specific wavelengths, a 270 fs decay time for the ground state recovery was observed in plastocyanin from Synechococcus PCC7942 when excited with a 33 fs pulse at $635 \mathrm{~nm}$ [18], and also in Pseudomonas aeruginosa azurin [14] and poplar plastocyanin [15] by exciting the sample with a $10 \mathrm{fs}$ pulse centered at $550 \mathrm{~nm}$ (not exactly in resonance with the LMCT bands which instead show the maximum at around 630 and $600 \mathrm{~nm}$ for azurin and plastocyanin, respectively) and analyzing the differential transmission at 580 and $560 \mathrm{~nm}$ in the case of azurin and plastocyanin, respectively. Some of the authors investigated the dynamics of the LMCT transition in umecyanin from Horseradish root upon excitation with 15 -fs pulses in resonance with LMCT band and detection at several wavelengths. A ground-state recovery with wavelength-dependent time constants, from 470 to $700 \mathrm{fs}$, showing a maximum at the absorption peak of the LMCT band was observed along with shorter time constants at both the blue and the red wings of the LMCT band [16]. Pump-probe measurements of rusticyanin excited state transition upon excitation with $25 \mathrm{fs}$ pulse tuned to the maximum of the LMCT band have been recently reported, and a ground state recovery with a time constant of about $230 \mathrm{fs}$ has been observed for all the investigated probe wavelengths [17]. Therefore, POXC excited state deactivation rate is lower than that of the other single-step deactivation processes occurring in copper proteins except for the umecyanin. The excited state deactivation rate in LMCT T1 Cu site transitions has been connected to the energy gap between the involved ligand and metal orbitals, and an inverse dependence of the deactivation rate on the gap has been proposed [16]. Accordingly, the longer decay time observed for POXC could be an indication of a coupling between the $\mathrm{Cu}-\mathrm{S}$ and the $\mathrm{d}-\mathrm{d}$ transitions slightly altered with respect to all the investigated $\mathrm{T} 1 \mathrm{Cu}$ site-containing proteins. The characteristics observed for the excited-state deactivation process occurring in POXC suggest that its $\mathrm{T} 1 \mathrm{Cu}$ site characteristics are different from those of the other proteins investigated by ultrafast resonant pump-probe spectroscopy. This well correlates with the hypothesis that POXC T1 Cu is strongly coordinated to only the three ligands constituting the common T1 Cu ligand set, i.e., thiolate sulfur of a cysteine residue and the imidazole nitrogens of two histidine residues, and thus T1 site features a trigonal geometry $[3,10,28]$. In fact in all of the copper-containing proteins so far investigated by ultrafast resonant pump-probe spectroscopy the T1 copper is ligated to at least four ligands. In particular in plastocyanins and in rusticyanin the copper atom is coordinated to two histidines and one cysteine residue and also to a sulfur atom of a methionine, leading to a tetragonal T1 site geometry. In azurins the sulfur atom of a methionine is the first axial ligand and the backbone carbonyl oxygen of a glycine residue provides a second weak axial interaction resulting in a trigonal bipyramidal geometry. In umecyanin the presence of a single strong axial ligand (a Gly) as fourth ligand leads to a distorted tetrahedral geometry of the T1 Cu site. 

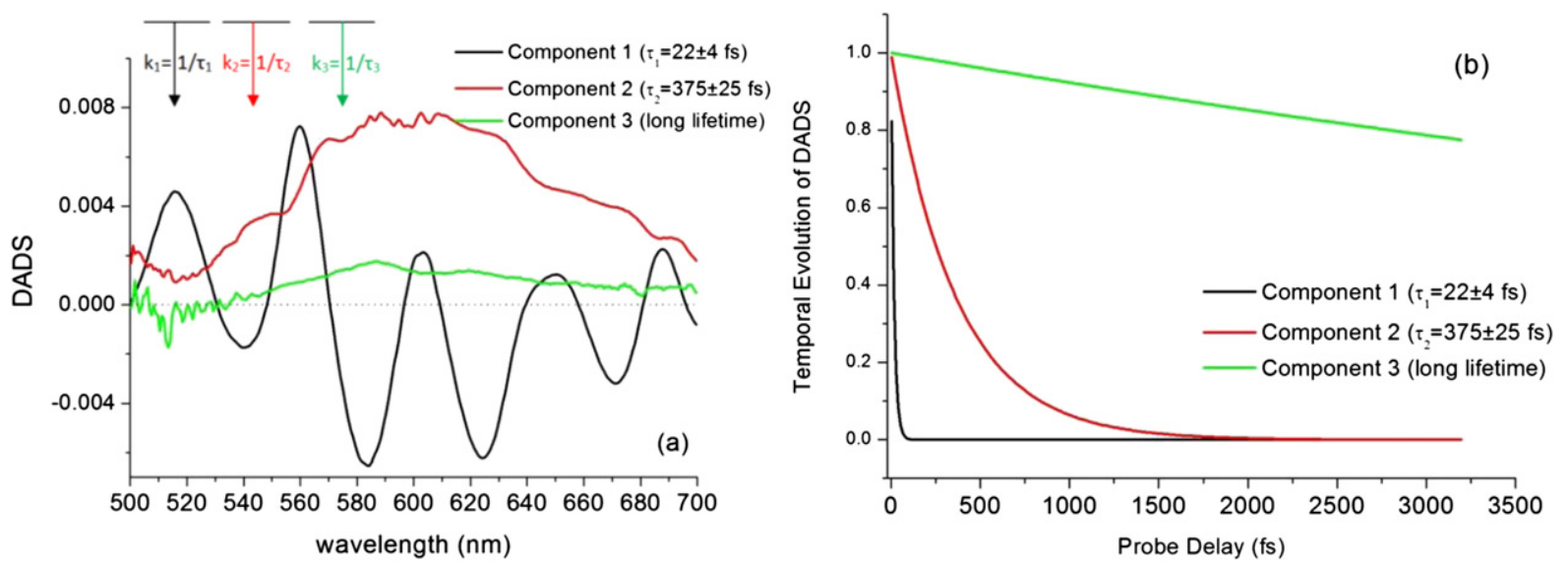

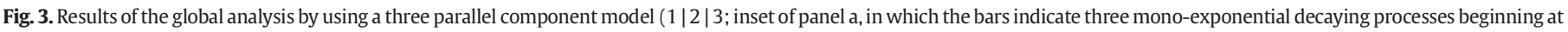

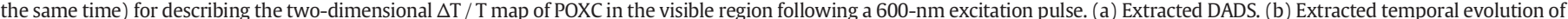

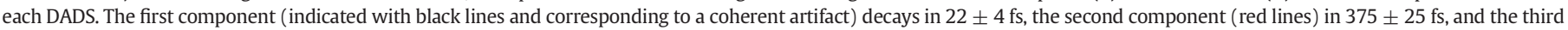
one (green lines) decays in more than $9 \mathrm{ps}$.

In order to analyze the oscillatory component superimposed on the previously presented decaying signal, we preliminarily subtracted the $2 \mathrm{D} \Delta \mathrm{T} / \mathrm{T}$ map obtained by global analysis from the bare signal and we then considered the residuals. In Fig. 4a a 2D map of the residuals as obtained from the data reported in Fig. 2a is shown, along with the residual traces for selected probe wavelengths (Fig. 4b).

Oscillations around the zero level displaying a complex wavelength dependence both in amplitude and in phase are observed, as confirmed also by the time traces considered. Residuals were then analyzed by performing a Fourier Transform (FT) to extract the frequencies of the single components. Pump-probe spectroscopy allows the observation

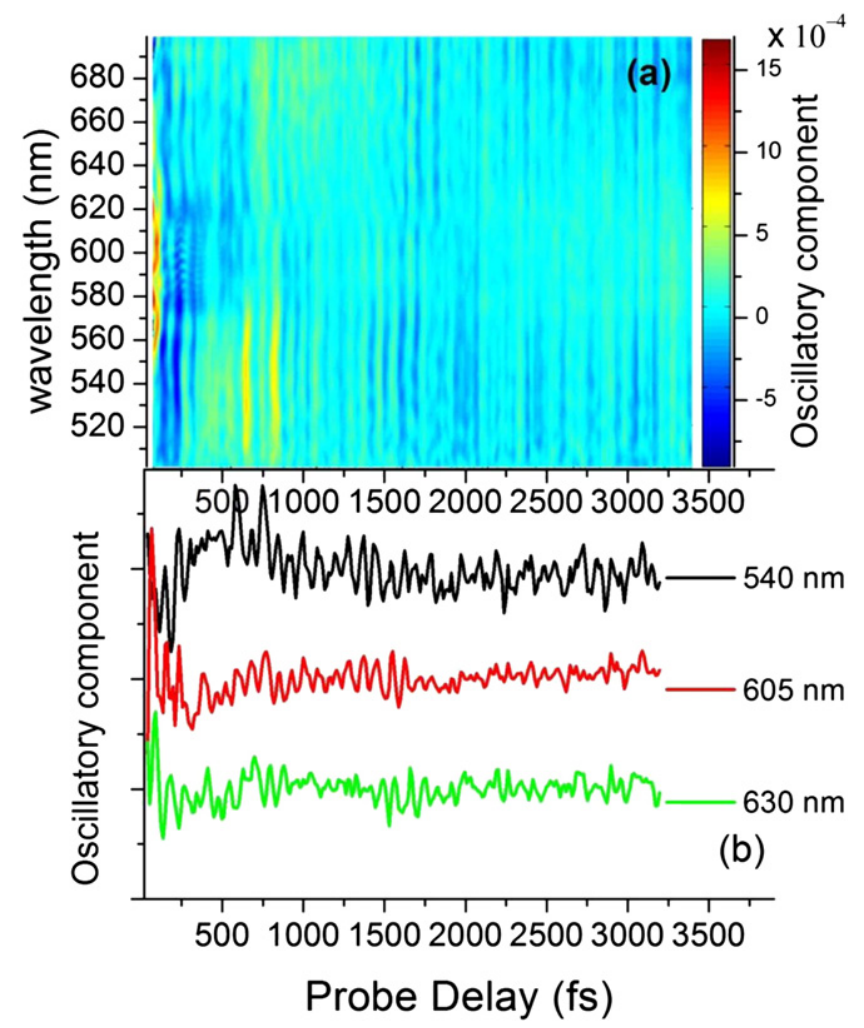

Fig. 4. (a) $2 \mathrm{D}$ map of the oscillatory component of the signals as obtained from the $\Delta T / T$ map after subtraction of the slowly decaying signal. (b) Oscillatory component traces extracted at specific probe wavelengths. of vibrational coherences in the time domain, well complementing conventional frequency-domain Raman spectroscopy (vide infra). The FT intensity spectra as obtained for all the investigated probe wavelengths are reported as a FT intensity map in Fig. 5a. The most prominent modes are located in the $350-450 \mathrm{~cm}^{-1}$ region, with two intense peaks detected at around 380 and $430 \mathrm{~cm}^{-1}$ and a shoulder at around $350 \mathrm{~cm}^{-1}$. Notably, the FT intensity is highly dependent on the probe wavelength, suggesting a different origin of the single modes. In particular, the modes in the $350-450 \mathrm{~cm}^{-1}$ region are more intense in the 500$580 \mathrm{~nm}$ probe wavelength range even with maxima and minima in the intensity. All these features are further confirmed by the inspection of the FT intensity spectra extracted at specific probe wavelengths shown in Fig. 5b where the main observed peaks are labeled. The measured coherent oscillations could be assigned to vibrational wavepackets motion on the ground and/or the excited state PES [27]. Pump-probe spectroscopy involves a three-field interaction with the sample; two of these with the pump, creating a population, and one with the probe, which then interrogates it. For short pump pulses, two fields in the pump pulse can excite, in phase, several vibrational eigenstates of the excited state, creating a coherent superposition in the form of a population wave-packet oscillating on the excited state $\mathrm{PES}$, leaving and returning to the Frank-Condon region. Another possibility is that the first field induces a polarization wave-packet on the excited state PES, which then propagates for some time during the pump pulse so that the second interaction with the pump field brings the wave-packet back down to the ground state, displaced from the equilibrium position. In this way, ground state oscillations are generated by a mechanism known as resonant impulsive stimulated Raman scattering (RISRS) [29].

Assignment of the observed modes to the ground or excited state is critical and is based on a comparison with steady state RR spectrum, and on oscillation lifetime. If we consider both these elements, we can assign the prominent observed oscillations (mainly, the 310-, 385-, 430- and $441-\mathrm{cm}^{-1}$ modes) to the ground state. In fact, these modes have counterparts in the RR spectrum (to be discussed in the following) and the oscillations are found to persist for a time significantly longer than the 375 -fs excited state lifetime. The assignment of the other modes is not straightforward.

The RR spectrum of laccase from $P$. ostreatus, which is not available in literature, is shown in Fig. 6 for the range $300-500 \mathrm{~cm}^{-1}$. The spectrum was obtained from POXC in aqueous solution at the same concentration used in the pump-probe experiments with excitation at $632.8 \mathrm{~nm}$ almost in resonance with the LMCT band. Even if the spectrum is rather noisy, some clear peaks can be evidenced. In particular, intense peaks 


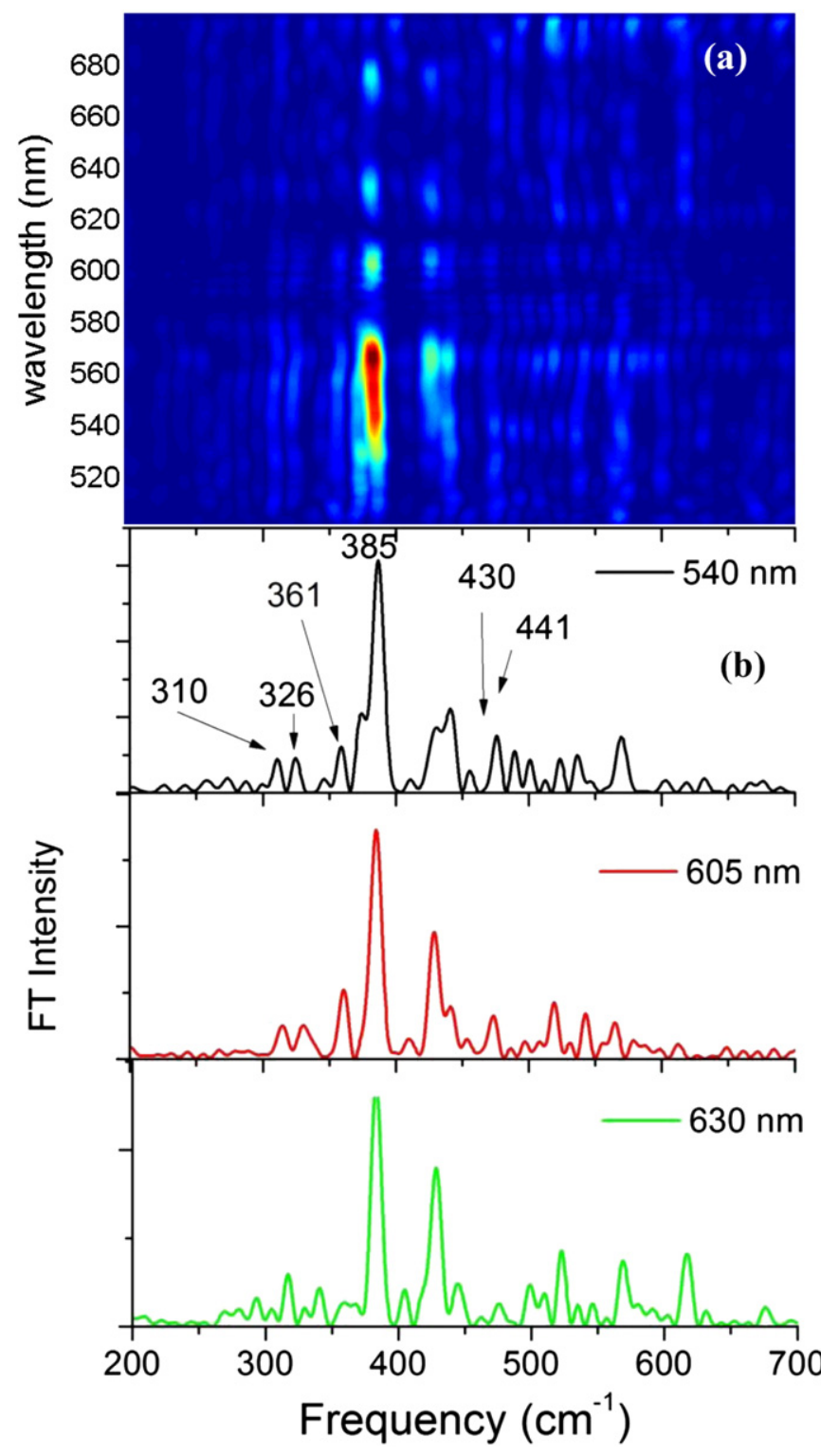

Fig. 5. (a) 2D map of the Fourier intensity of the oscillatory component shown in Fig. 4a (see text). (b) Fourier Intensity spectra extracted at specific probe wavelengths. Main peaks are labeled.

at $372,382,393,404$ and $414 \mathrm{~cm}^{-1}$ together with some shoulders around 355,427 and $434 \mathrm{~cm}^{-1}$ can be observed. The modes around $400 \mathrm{~cm}^{-1}$, which constitute in general the fingerprint of the RR spectra of $\mathrm{T} 1$ copper sites, can be assigned to the mixing of the $\mathrm{Cu}-\mathrm{S}$ (Cys) stretching vibration with multiple heavy atom bending modes of the ligand and adjacent residues [30-33]. POXC RR vibrational features are rather similar to those observed in some laccases and blue copper proteins, characterized by similar organization of the copper active site [30-34]. Slight modulations in the positions of the main peaks could be related to differences in the T1 copper site as well as in the surrounding protein matrix. From a comparison of RR spectrum with the FT spectra shown in Fig. 5, it comes out that almost all the vibrational features observed in the RR spectrum find a very good correspondence with some of the peaks emerging from the pump-probe experiment. However, the FT spectra have a higher richness in the spectral content with respect to those from RR spectroscopy, as also observed in other cases [12, 14-17]. Additionally, the slight shift measured in the positions of the pump-probe peaks with respect to that of the conventional RR is similar to that observed in other studies [12,14-17].

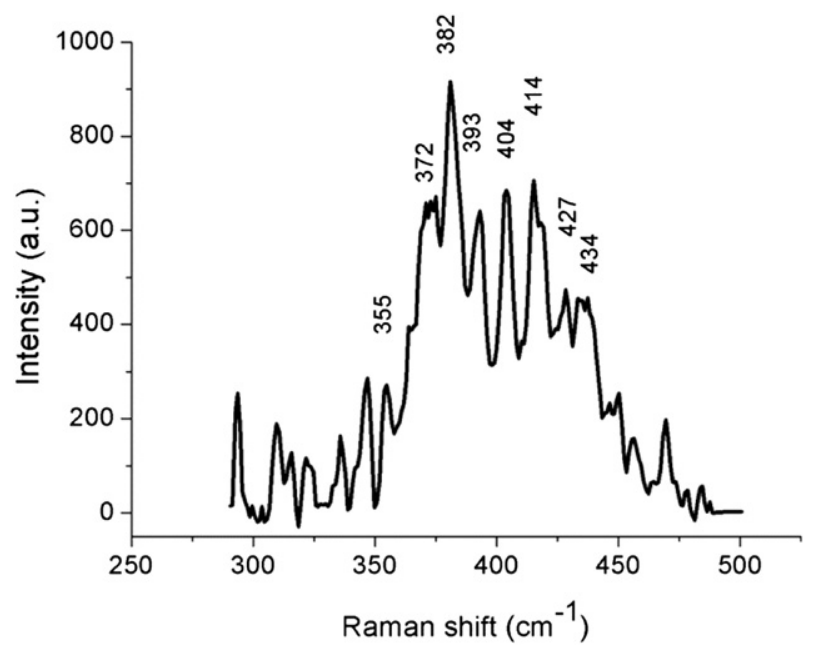

Fig. 6. Resonance Raman spectrum of $\mathrm{Cu}(\mathrm{II}) \mathrm{POXC}$ obtained with an excitation of $632.8 \mathrm{~nm}$.

\section{Conclusions}

In this paper, we have presented an ultrafast spectroscopy study of the dynamics of the initial step of the complex charge transfer process occurring in laccase from $P$. ostreatus. Upon excitation with $15 \mathrm{fs}$ pulses resonant with the LMCT transition we observe a single step groundstate recovery with a 375 fs time constant. This evidences a decaying process similar to those occurring in some cupredoxins, although with a peculiar lifetime. The unusual time constant characterizing the MLCT transition in POXC is discussed in connection with the peculiarities of the enzyme, since this study is the only one reporting on the characterization of the light-induced CT dynamics in a trigonal T1 Cu site. Coupled to the population dynamics, we observe vibrational coherence induced by the ultrashort pump pulse. Vibrational activity is mainly seen in the ground state, as expected in a system with fast excited-state decay. These results give a detailed description of the deactivation processes following a light induced charge transfer mimicking the first step of the naturally relevant charge transfer chain occurring in a fungal laccase. This opens the route for the investigation, by the same approach, of the whole charge transfer process, which begins at the T1 $\mathrm{Cu}$, where the four single electron oxidations of the reducing substrate occur, and then involves the trinuclear copper site where the $\mathrm{O}_{2}$ is finally reduced to water. The description of the whole process is of crucial importance from both fundamental and technological standpoints. In fact, laccases are almost ubiquitary enzymes (they have been isolated from plants, from some kinds of bacteria, and from insects too) carrying out a variety of physiological roles during their life cycle, including morphogenesis, fungal plant pathogen/host interaction, stress defense, and lignin degradation. In addition, they have an important role in the synthesis of some biopolymers, especially phenolic biopolymers, and are also able to catalyze the oxidation of other substituted polyphenols, aromatic amines, benzenethiols and a series of other compounds.

\section{Acknowledgments}

The authors wish to thank Dr. Cinzia Pezzella and Dr. Alessandra Piscitelli from University of Napoli "Federico II" for their valuable help. G. C. acknowledges support by the European Research Council Advanced Grant STRATUS (ERC-2011-AdG No. 291198).

\section{References}

[1] P. Giardina, V. Faraco, C. Pezzella, A. Piscitelli, S. Vanhulle, G. Sannia, Laccases: a never-ending story, Cell. Mol. Life Sci. 67 (2010) 369-385.

[2] E.I. Solomon, A.J. Augustine, J. Yoon, $\mathrm{O}_{2}$ reduction to $\mathrm{H}_{2} \mathrm{O}$ by the multicopper oxidases, Dalton Trans. 30 (2008) 3921-3932. 
[3] L. Quintanar, C. Stoj, A.B. Taylor, P.J. Hart, D.J. Kosman, E.I. Solomon, Shall we dance? How a multicopper oxidase chooses its electron transfer partner, Acc. Chem. Res. 40 (2007) 445-452.

[4] E.I. Solomon, U.M. Sundaram, T.E. Machonkin, Multicopper oxidases and oxygenases, Chem. Rev. 96 (1996) 2563-2605.

[5] A. Messerschmidt, R. Huber, The blue oxidases, ascorbate oxidase, laccase and ceruloplasmin. Modelling and structural relationships, Eur. J. Biochem. 187 (1990) 341-352.

[6] U.A. Germann, G. Muller, P.E. Hunziker, K. Lerch, Characterization of two allelic forms of Neurospora crassa laccase, J. Biol. Chem. 263 (1988) 885-896.

[7] A.E. Palmer, D.W. Randall, F. Xu, E.I. Solomon, Spectroscopic studies and electronic structure description of the high potential type 1 copper site in fungal laccase: insight into the effect of the axial ligand, J. Am. Chem. Soc. 121 (1999) 7138-7149, http://dx.doi.org/10.1021/ja991087v.

[8] K. Nakamura, N. Go, Function and molecular evolution of multicopper blue proteins, Cell. Mol. Life Sci. 62 (2005) 2050-2066

[9] M.T. Cambria, D. Gullotto, S. Garavaglia, A. Cambria, In silico study of structural determinants modulating the redox potential of Rigidoporus lignosus and other funga laccases, J. Biomol. Struct. Dyn. 30 (2012) 89-101, http://dx.doi.org/10.1080/ 07391102.2012.674275.

[10] C. Dennison, Investigating the structure and function of cupredoxin, Coord. Chem. Rev. 249 (2005) 3025-3054.

[11] M.D. Edington, W.M. Diffey, W.J. Doria, R.E. Riter, W.F. Beck, Radiationless decay from the ligand-to-metal charge-transfer state in the blue copper protein plastocyanin, Chem. Phys. Lett. 275 (1997) 119-126.

[12] F. Gruia, M. Kubo, X. Ye, D. Ionascu, C. Lu, R.K. Poole, S.-R. Yeh, P.M. Champion, Coherence spectroscopy investigations of the low-frequency vibrations of heme: effects of protein-specific perturbations, J. Am. Chem. Soc. 130 (2008) 5231-5244.

13] F. Gruia, D. Ionascu, M. Kubo, X. Ye, J. Dawson, R.L. Osborne, S.G. Sligar, I. Denisov, A. Das, T.L. Poulos, J. Terner, P.M. Champion, Low-frequency dynamics of caldariomyces fumago chloroperoxidase probed by femtosecond coherence spectroscopy, Biochemistry 47 (2008) 5156-5167.

[14] T. Cimei, A.R. Bizzarri, S. Cannistraro, G. Cerullo, S. De Silvestri, Vibrational coherence in Azurin with impulsive excitation of the LMCT absorption band, Chem. Phys. Lett. 362 (2002) 497-503.

[15] T. Cimei, A.R. Bizzarri, G. Cerullo, S. De Silvestri, S. Cannistraro, Excited state chargetransfer dynamics study of poplar plastocyanin by ultrafast pump-probe spectroscopy and molecular dynamics simulation, Biophys. Chem. 106 (2003) 221-231.

[16] I. Delfino, C. Manzoni, K. Sato, C. Dennison, G. Cerullo, S. Cannistraro, Ultrafast pump-probe study of excited-state charge-transfer dynamics in umecyanin from horseradish root, J. Phys. Chem. B 110 (2006) 17252-17259.

[17] A.R. Bizzarri, D. Brida, S. Santini, G. Cerullo, S. Cannistraro, Ultrafast pump-probe study of the excited-state charge-transfer dynamics in blue copper rusticyanin, J. Phys. Chem. B 116 (2012) 4192-4198, http://dx.doi.org/10.1021/jp301484g.

[18] S. Nakashima, Y. Nagasawa, K. Seike, T. Okada, M. Sato, T. Kohzuma, Coherent dynamics in ultrafast charge-transfer reaction of plastocyanin, Chem. Phys. Lett. 331 (2000) 396-402.

[19] Y. Nagasawa, K. Fujita, T. Katayama, Y. Ishibashi, H. Miyasaka, T. Takabe, S. Nagao, S. Hirota, Coherent dynamics and ultrafast excited state relaxation of blue copper protein; plastocyanin, Phys. Chem. Chem. Phys. 12 (2010) 6067-6075.
[20] L.D. Book, D.C. Arnett, H. Hu, N.F. Scherer, Ultrafast pump-probe studies of excitedstate charge-transfer dynamics in blue copper proteins, J. Phys. Chem. A 102 (1998) 4350-4359.

[21] F. Autore, C. Del Vecchio, F. Fraternali, P. Giardina, G. Sannia, V. Faraco, Molecular determinants of peculiar properties of a Pleurotus ostreatus laccase: analysis by sitedirected mutagenesis, Enzyme Microb. Technol. 45 (2009) 507-513.

[22] C. Pezzella, M.E. Russo, A. Marzocchella, P. Salatino, G. Sannia, Immobilization of a Pleurotus ostreatus laccase mixture on perlite and its application to dye decolourisation, Biomed. Res. Intern. 2014 (2014) (Article ID 308613, 11 pp. http://dx.doi.org/10.1155/2014/308613).

[23] C. Manzoni, D. Polli, G. Cerullo, Two-color pump-probe system broadly tunable over the visible and the near infrared with sub-30-fs resolution, Rev. Sci. Instrum. 77 (2006) 023103/1-023103/9.

[24] M. Zavelani-Rossi, G. Cerullo, S. De Silvestri, L. Gallmann, N. Matuschek, G. Steinmeyer, U. Keller, G. Angelow, V. Scheuer, T. Tschudi, Pulse compression over 170-THz bandwidth in the visible using only chirped mirrors, Opt. Lett. 26 (2001) 1155-1157.

[25] I.H. van Stokkum, D.S. Larsen, R. van Grondelle, Global and target analysis of time resolved spectra, Biochim. Biophys. Acta 1657 (2004) 82-104.

[26] I. Delfino, G. Cerullo, S. Cannistraro, C. Manzoni, D. Polli, C. Dapper, W.E. Newton, Y. Guo, S.P. Cramer, Observation of terahertz vibrations in the nitrogenase femo cofactor by femtosecond pump-probe spectroscopy, Angew. Chem. Int. Ed. 49 (2010) 3912-3915.

[27] W.T. Pollard, S.L. Dexheimer, Q. Wang, L.A. Peteanu, C.V. Shank, R.A.J. Mathies, Phys. Chem. 96 (1992) 6147-6158.

[28] E.I. Solomon, K.W. Penfield, A.A. Gewirth, M.D. Lowery, S.E. Shadle, J.A. Guckert, L.B. LaCroix, Electronic structure of the oxidized and reduced blue copper sites: contributions to the electron-transfer pathway, reduction potential, and geometry, Inorg. Chim. Acta 243 (1996) 67-78.

[29] S. Ruhman, B. Kohler, A.G. Joly, K.A. Nelson, Intermolecular vibrational motion in $\mathrm{CS}_{2}$ liquid at $165<\mathrm{T}<300 \mathrm{~K}$ observed in femtosecond time-resolved impulsive stimulated scattering, Chem. Phys. Lett. 141 (1987) 16-24.

[30] G.R. Loppnow, E. Fraga, Proteins as solvents: the role of amino acid composition in the excited-state charge transfer dynamics of plastocyanins, J. Am. Chem. Soc. 119 (1997) 896-905.

[31] T.J. Thamann, P. Franko, LJ Willis, T.M. Loehr, Normal coordinate analysis of the copper center of azurin and the assignment of its resonance Raman spectrum, Proc. Natl. Acad. Sci. U. S. A. 79 (1982) 6396-6400.

[32] J. Han, E.T. Adman, T. Beppu, R. Codd, H.C. Freeman, L. Huq, T.M. Loehr, J. SandersLoehr, Resonance Raman spectra of plastocyanin and pseudoazurin: evidence for conserved cysteine ligand conformations in cupredoxins (blue copper proteins), Biochemistry 30 (1991) 10904-10913.

[33] I. Delfino, K. Sato, M.D. Harrison, L. Andolfi, A.R. Bizzarri, C. Dennison, S. Cannistraro, Optical spectroscopic investigation of the alkaline transition in umecyanin from horseradish root, Biochemistry (US) 44 (2005) 16090-16097.

[34] E.I. Solomon, R.G. Hadt, Recent advances in understanding blue copper proteins, Coord. Chem. Rev. 255 (2011) 774-789. 